\section{Numerical investigation of the drag force reduction for homogeneous column of vehicles}

\author{
Władysław Hamiga, Wojciech Ciesielka \\ AGH University of Science and Technology, Poland
}

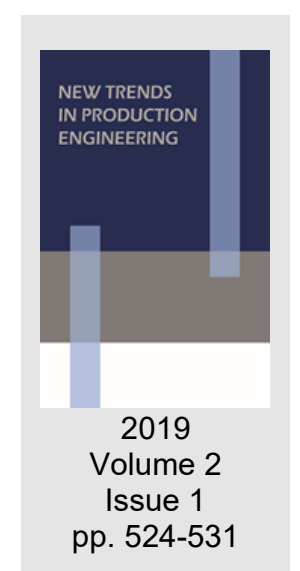

Date of submission to the Editor: 05/2019

Date of acceptance by the Editor: 08/2019

\title{
INTRODUCTION
}

A growing number of vehicles encouraged to look for alternative methods of reducing emission of harmful substances generated by road transport. The main pollutants arising from the combustion of fuel include hydrocarbons, nitrogen oxides, carbon monoxide, particulate matters (PM), aldehydes and carbon dioxide. However the most significant part of exhaust is carbon dioxide, which remain between 12-14\%. Analyzing statistical data from 2014 (Birol, 2016) and 2015 (Birol, 2017), the amount of carbon dioxide generated by road vehicles is the second largest cause of $\mathrm{CO}_{2}$ emissions from fuel combustion in the world and accounts for $20 \%$ of total emissions. One of the solutions leading to reducing the negative impact of transport on the environment is the use of aerodynamic wake located behind moving vehicles. Creating organized columns of vehicle (convoys) allows to reduce the drag force, and at the same time to decrease amount of fuel combustion and reduce emission of harmful substances.

Zabat et al. investigated aerodynamic forces for homogeneous columns created from two to four passenger cars. The experimental study were carried out in a wind tunnel. The length of the single car was 0.6 meters, and the distance between the vehicles came from the 0 to 3 length of the car (Zabat et al., 1994). Schito et al. conducted research for a homogeneous column consisting of one to six vehicles. The length of the single car was 4 meters. The searching result was the drag force coefficient. The test was carried out for three different distances between vehicles: 2, 3 and 4 meters (Schito et al., 2012).

Le Good et al. performed experimental studies on a scale for a homogeneous column consisting of 2 to 5 passenger cars. At sufficiently small distances between vehicles it was observed an unfavorable effect that is the increase of drag force (Le Good et al 2018).

A few works are also focused on the columns consisting only of heavy duty vehicles (Browand, 2004, Humphreys, 2016, Siemon and Nichols, 2018, ohra, 2018). 
In this work, object of the research is a homogeneous column which consists of van type vehicles. These kind of vehicles have been widely used in transport and constitute an important link in land logistics. The purpose of this work is to develop a numerical model which allows to determine aerodynamic forces on individual vehicles in the column.

\section{METHODOLOGY OF RESEARCH}

The problem of studying phenomena around a moving vehicle column is very complex and therefore, in order to explore it, the use of appropriate tools are required. The use of computer simulation allows for the reconstruction of complex phenomena related to fluid flow (Computational Fluid Dynamics CFD). At the initial stage CFD allows to reduce costs and time of analysis in comparison to experimental research. It leads to quick and efficient optimization of the issue. To solve this problem, the Finite Volume Method was implemented in the ANSYS Fluent package. The drag force obtained from the numerical calculations was converted into a dimensionless force coefficient of air resistance based on dependence (1).

$$
\mathrm{C}_{\mathrm{d}}=\frac{2 \mathrm{~F}_{\mathrm{d}}}{\rho_{\mathrm{p}} A v^{2}}
$$

where:

$\mathrm{F}_{\mathrm{d}}-$ drag force $[\mathrm{N}]$

$\rho_{\mathrm{p}}-$ air density $\left[\mathrm{kg} \cdot \mathrm{m}^{-3}\right]$

A - frontal area of the vehicle $\left[\mathrm{m}^{-2}\right]$

$\mathrm{v}-$ relative fluid velocity $\left[\mathrm{m} \cdot \mathrm{s}^{-1}\right]$

\section{Mathematical model}

To describe the phenomenon of air flow around the column of vehicle, specific air properties and simplifications were adopted. It was assumed that the fluid is incompressible, viscous and Newtonian, there are no external forces (gravitational interactions are omitted), and the solution does not depend on time. In developing those assumptions, the continuity equation (2) and the equation of momentum transport (3-5) are valid in the studied area, in the following form:

$$
\begin{array}{r}
\frac{\partial \overline{u_{x}}}{\partial x}+\frac{\partial \overline{u_{y}}}{\partial y}+\frac{\partial \overline{u_{z}}}{\partial z}=0 \\
\rho_{p}\left(u_{x} \frac{\partial \overline{u_{x}}}{\partial x}+u_{y} \frac{\partial \overline{u_{x}}}{\partial y}+u_{z} \frac{\partial \overline{u_{x}}}{\partial z}\right) \\
=-\frac{\partial p}{\partial x}+\mu_{p}\left(\frac{\partial^{2} \overline{u_{x}}}{\partial x^{2}}+\frac{\partial^{2} \overline{u_{x}}}{\partial y^{2}}+\frac{\partial^{2} \overline{u_{x}}}{\partial z^{2}}\right) \\
-\rho_{p}\left(\frac{\partial \overline{u_{x}^{\prime} u_{x}^{\prime}}}{\partial x}+\frac{\partial \overline{u_{x}^{\prime} u_{y}^{\prime}}}{\partial y}+\frac{\partial \overline{u_{x}^{\prime} u_{z}^{\prime}}}{\partial z}\right)
\end{array}
$$




$$
\begin{aligned}
\rho_{p}\left(u_{x} \frac{\partial \overline{u_{y}}}{\partial x}+\right. & \left.u_{y} \frac{\partial \overline{u_{y}}}{\partial y}+u_{z} \frac{\partial \overline{u_{y}}}{\partial z}\right) \\
= & -\frac{\partial p}{\partial y}+\mu_{p}\left(\frac{\partial^{2} \overline{u_{y}}}{\partial x^{2}}+\frac{\partial^{2} \overline{u_{y}}}{\partial y^{2}}+\frac{\partial^{2} \overline{u_{y}}}{\partial z^{2}}\right) \\
& -\rho_{p}\left(\frac{\partial \overline{u_{y}^{\prime} u_{x}^{\prime}}}{\partial x}+\frac{\partial \overline{u_{y}^{\prime} u_{y}^{\prime}}}{\partial y}+\frac{\partial \overline{u_{y}^{\prime} u_{z}^{\prime}}}{\partial z}\right) \\
\rho_{p}\left(u_{x} \frac{\partial \overline{u_{z}}}{\partial x}+\right. & \left.u_{y} \frac{\partial \overline{u_{z}}}{\partial y}+u_{z} \frac{\partial \overline{u_{z}}}{\partial z}\right) \\
= & -\frac{\partial p}{\partial z}+\mu_{p}\left(\frac{\partial^{2} \overline{u_{z}}}{\partial x^{2}}+\frac{\partial^{2} \overline{u_{z}}}{\partial y^{2}}+\frac{\partial^{2} \overline{u_{z}}}{\partial z^{2}}\right) \\
& -\rho_{p}\left(\frac{\partial \overline{u_{z}^{\prime} u_{x}^{\prime}}}{\partial x}+\frac{\partial \overline{u_{z}^{\prime} u_{y}^{\prime}}}{\partial y}+\frac{\partial \overline{u_{z}^{\prime} u_{z}^{\prime}}}{\partial z}\right)
\end{aligned}
$$

where:

$\bar{u}_{x}, \bar{u}_{y}, \bar{u}_{z}$ - average values of velocity vector components,

$u_{x}^{\prime}, u_{y}^{\prime}, u_{z}^{\prime}$ - fluctuations of velocity vector components,

$p$ - pressure,

$\rho_{p}-$ air density,

$\mu_{p}-$ air dynamic viscosity.

The unknown parameters are components of air velocity and pressure distribution, which will be used to determine the drag force on vehicles.

\section{Validation}

The development of the numerical model of a homogeneous column requires the selection of appropriate simulation parameters, such as the turbulence model, the height of the first cell above the vehicle surface $(y+$ parameter), the number of layers in the boundary layer and the mesh density. For this purpose, verification calculations were carried out using reference structure - Ahmed body. For the geometry of Ahmed body, it was used the model of turbulence kw SST, parameter y+ equal 30 (Ciesielka and Hamiga, 2018, Simmonds, 2017) and the number of layers in the boundary layer equal 15. The drag force coefficient was calculated for 9 different velocities from $20 \mathrm{~m} / \mathrm{s}$ till $100 \mathrm{~m} / \mathrm{s}$. Coefficients were compared with the studies obtained by (Bayraktar et al., 2001; Meile et al., 2010), whom achieved consistent results.

Obtained error was in the range of $1.39 \%$ to $8.17 \%$, depending on the velocity of airflow. The values of the drag force coefficient $\mathrm{Cd}$ are presented in Table 1.

Table 1 Drag coefficient for Ahmed body

\begin{tabular}{|c|c|c|c|c|c|c|c|c|c|}
\hline $\begin{array}{c}\text { Velocity } \\
{[\mathrm{m} / \mathrm{s}]}\end{array}$ & 20 & 30 & 40 & 50 & 60 & 70 & 80 & 90 & 100 \\
\hline $\mathrm{Cd} \mathrm{[-]}$ & 0.333 & 0.314 & 0.302 & 0.297 & 0.294 & 0.293 & 0.305 & 0.304 & 0.304 \\
\hline Error [\%] & 3.85 & 2.11 & 1.39 & 1.81 & 2.28 & 2.86 & 7.74 & 7.85 & 8.17 \\
\hline
\end{tabular}




\section{Numerical model - van Geometry model}

The investigated vehicles are represented by van type geometry (Fig. 1), with general dimensions of $2.5 \times 2.05 \times 6$ meters.
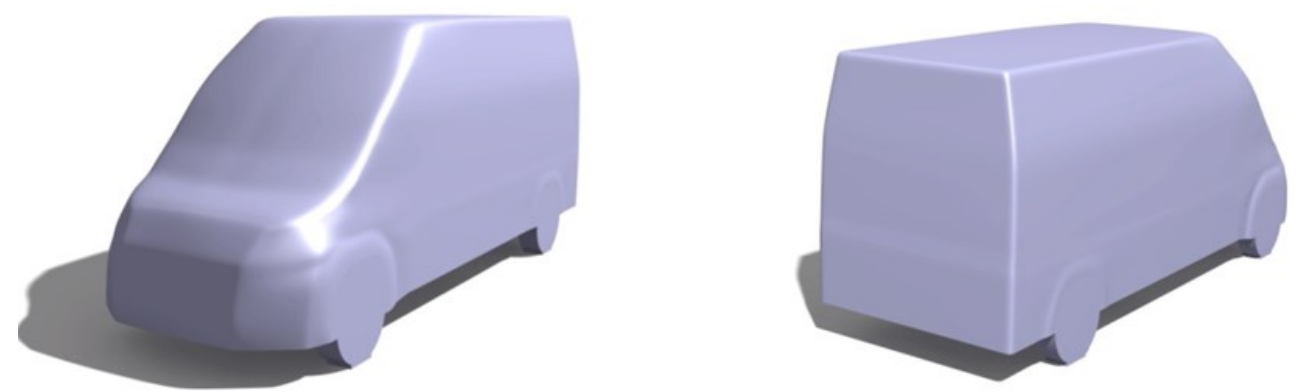

Fig. 1 Isometric view for van vehicle - front and rear view

The shape of the body was detailed in accordance with the geometry of the Ford Transit model. In order to reduce the calculation time, some simplifications were adopted. The influence of the mirrors,

wipers, antennas, free space between the wheel and wheel arches was neglected, and the chassis of the vehicle was simplified. The cars were organized in columns consisting of three vehicles in the row. For created columns, the expected value is the air drag force depending on the distance between cars. The aerodynamic forces for single vehicle were also calculated. Geometric vehicle models were created using the surface modeling tool in the CATIA software.

\section{Discretization}

The geometric model of the domain has been discretized using a structural mesh (Fig. 2).

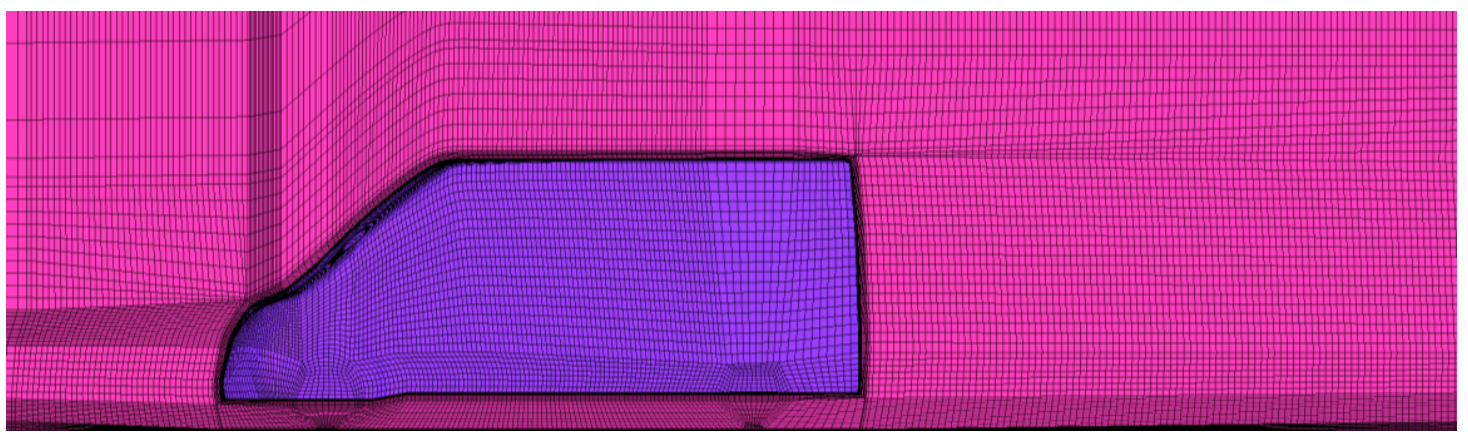

Fig. 2 Mesh around van at symmetry plane

The number of elements in the boundary layer around the geometry was 15 (the number of elements at height), and the height of the first element for the dimensionless $y+$ coefficient was equal to 30 . A further transition layer was used around the boundary layer to gradually pass between the cell sizes. The minimum value of the orthogonal quality parameter was 0.12 . Over $98 \%$ of the grid elements had a quality above 0.5 . The mesh independence has been investigated (Fig. 3). 


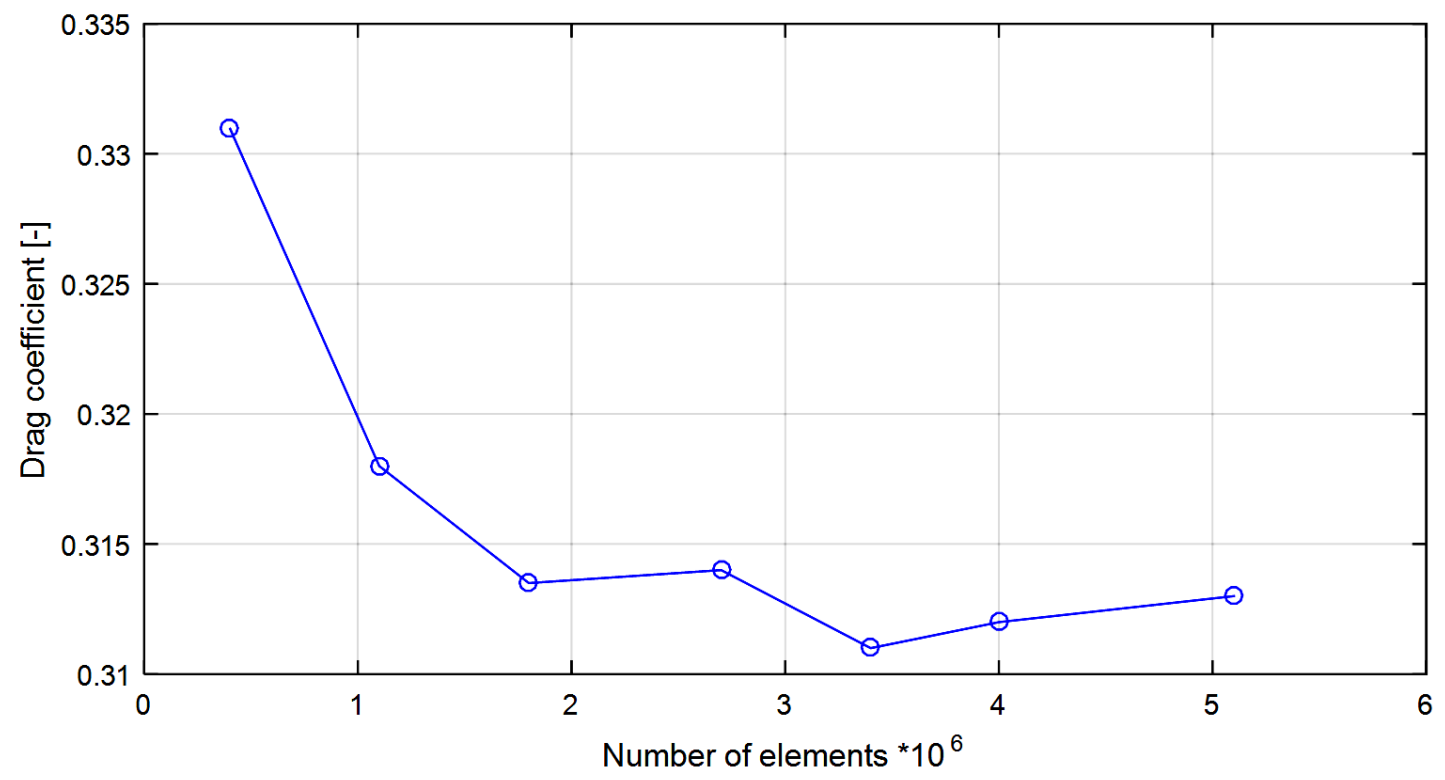

Fig. 3 Mesh independence. Number of elements versus drag coefficient

The number of elements in the model were between 0.4 to 5.1 million. Due to the reduction of computation time, the grid density was as small as possible. For further calculations, it was decided to use a grid corresponding to a density of 1.8 million elements, where the drag coefficient equal to 0.3135 .

\section{Boundary conditions}

A calculation area measuring $6 \times 12 \times 60$ meters was created around the geometry of van vehicles. In order to reduce the number of elements, only half of the domain was modeled and the condition of symmetry corresponding to zeroing of velocities and gradients was given. At the inlet to the computational area, an air velocity of $25 \mathrm{~m} / \mathrm{s}$ was adopted. A reference pressure of zero was applied to the outlet. Due to viscosity of the fluid, a velocity condition equal to zero was applied to the surface of the vehicles. In order to mirror movement of the road, the bottom surface was given a velocity equal to the inflow velocity (Fig. 4). In the boundary conditions, the rotating vehicle wheels are not included.

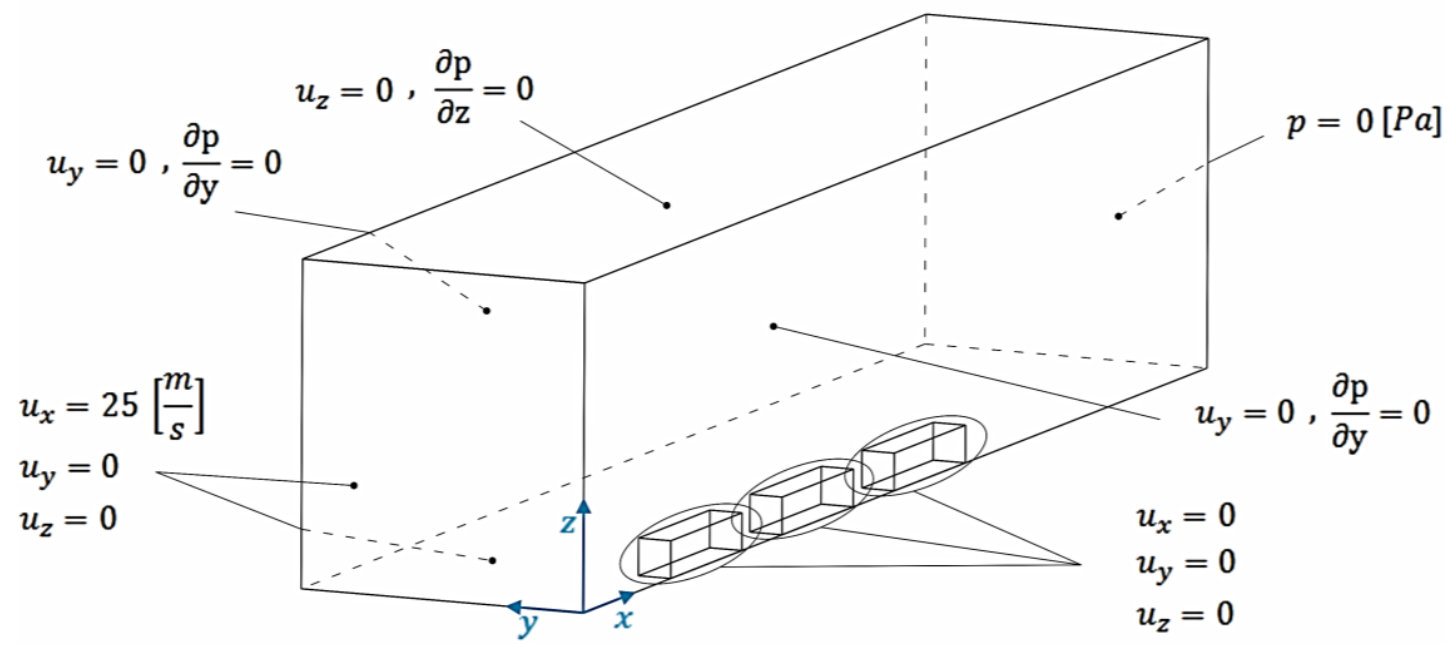

Fig. 4 Boundary conditions for homogeneous column of vans 


\section{RESULTS}

The studies were carried out for the steady state using the k- $\omega$ SST turbulence model. Simulation calculations were made for three different distances between vehicles: 2, 5 and 10 meters. For each configuration, the drag coefficient of individual cars was determined. The results are presented in Table 2, where Cd1 is a drag coefficient for the first van in row, Cd2 for the second van and Cd3 for the last one. Average drag coefficient for three vehicles was calculated and shown in last column.

Table 2 Drag coefficient of vans

\begin{tabular}{|c|c|c|c|c|}
\hline Spacing [m] & Cd1 [-] & Cd2 [-] & Cd3 [-] & Average [-] \\
\hline 2 & 0.225 & 0.240 & 0.305 & 0.257 \\
\hline 5 & 0.241 & 0.234 & 0.265 & 0.247 \\
\hline 10 & 0.288 & 0.236 & 0.246 & 0.257 \\
\hline
\end{tabular}

The reduction of operating force is observed on each vehicle, also for the one at the head of the column. Figures 5-7 show pressure distribution while maintaining three different spacing between vehicles. The legend expressed in pascal is shown in Figure 8. Significant differences in pressure distribution are mainly observed between vehicles. High pressure zones in these areas has positively influenced by the reduction of the drag force, especially for the first vehicle with a distance up to 5 meters. The distribution of the velocity field around vehicles are given in Figures 9-12.

Fig. 5 Pressure distribution at symmetry plane spacing between vans -2 meters

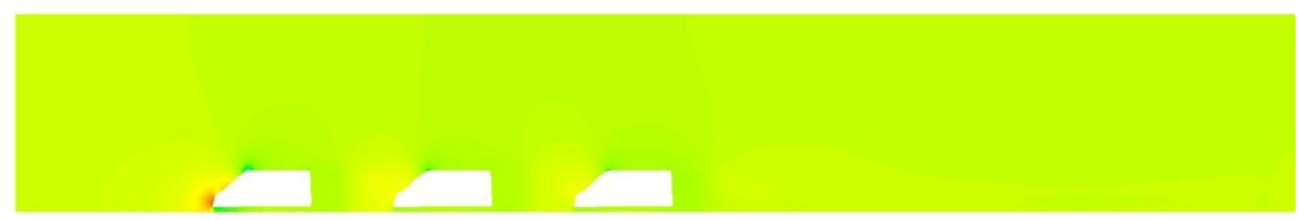

Fig. 6 Pressure distribution at symmetry plane spacing between vans -5 meters

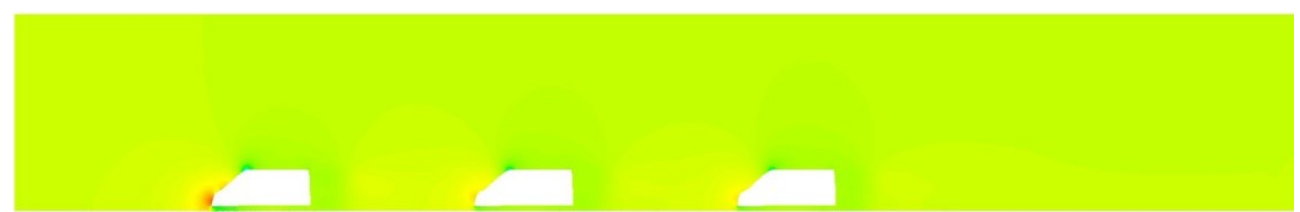

Fig. 7 Pressure distribution at symmetry plane spacing between vans -10 meters

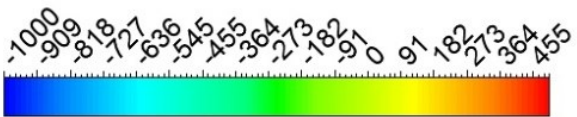

Fig. 8 Pressure distribution in pascal - legend 


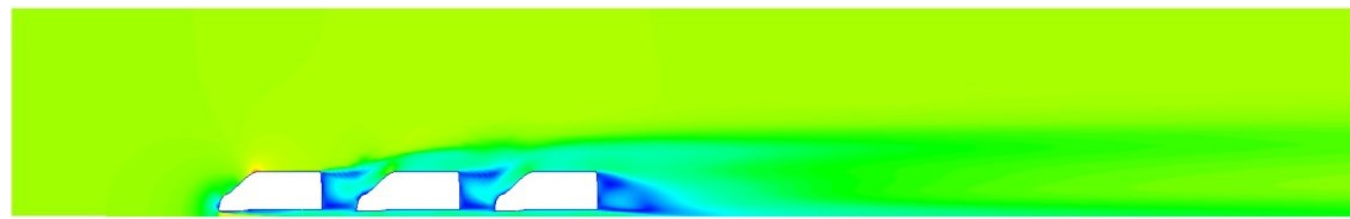

Fig. 9 Velocity distribution at symmetry plane spacing between vans -2 meters

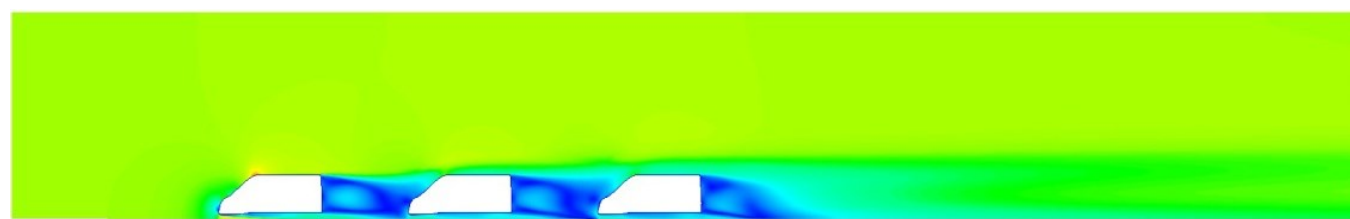

Fig. 10 Velocity distribution at symmetry plane spacing between vans -5 meters

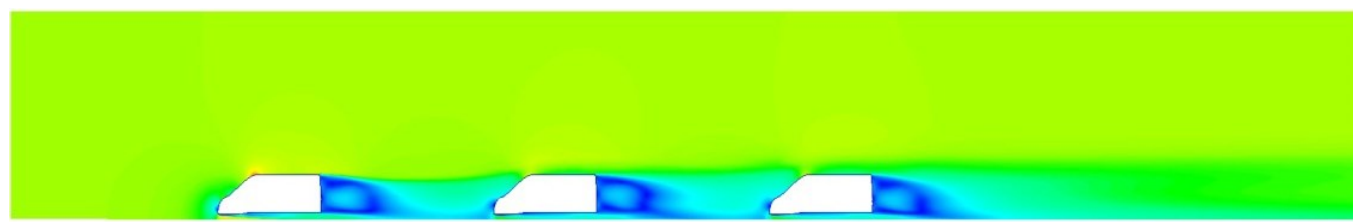

Fig. 11 Velocity distribution at symmetry plane spacing between vans -10 meters

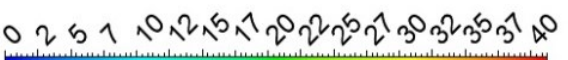

Fig. 12 Velocity distribution in meters per second - legend

Due to the flat shape of the rear of the car, the reduction of the air resistance force was significant even for a distance between vehicles up to 10 meters. Further research is required for a wider range of spacing and different value of velocity.

\section{CONCLUSION}

The developed numerical model of the moving column of vehicles allows to specify the acting of aerodynamic forces without performing expensive and timeconsuming experimental and operational tests. In order to verify the calculations, the reference geometry Ahmed body was used, and the results were compared. The coefficients calculated at simulation were consistent with results achieved in other studies.

Object of research was a homogeneous column consisting of van vehicles. The conducted simulation calculations prove the benefits of traveling in an organized column of vehicles. Each of the tested configurations showed a positive impact on the environment. Especially due to the reduction of the convoy's air drag force, and thus the reduction of the amount of harmful substances.

The main purpose of the work was to develop a numerical model for van vehicles. The model will be used in further studies for heterogeneous columns which are a wider issue.

Simulations were performed using the ANSYS Fluent software. The research was made in the ICM Computation Center on the Hydra computer, in CYFRONET on the ZEUS computer and on a desktop computer. The number 
of cores used for a single simulation ranged from 3 to 12 , and the reserved RAM from 12 to $48 \mathrm{~GB}$.

\title{
REFERENCES
}

Bayraktar, I., Landman, D., Baysal, O. (2001). Experimental and Computational Investigation of Ahmed Body for Ground Vehicle Aerodynamics. SAE Technical Paper 2001-01-2742.

Birol, F. (2016). $\mathrm{CO}_{2}$ emissions from fuel combustion, International Energy Agency.

Birol, F. (2017). $\mathrm{CO}_{2}$ emissions from fuel combustion, International Energy Agency.

Browand, F., McArthur, J., Radovich, C. (2004). Fuel Saving Achieved in the Field Test of Two Tandem Trucks. UC Berkeley: California Partners for Advanced Transportation Technology, pp. 1-28.

Ciesielka, W., Hamiga, W. (2018). Numerical modeling of airflow over column of vehicles using ANSYS $₫$ package, E3S Web of Conferences, 46, art. no. 00025, pp. 1-8.

Humphreys, H., Batterson, J., Bevly, D., Schubert, R. (2016). An Evaluation of the Fuel Economy Benefits of a Driver Assistive Truck Platooning Prototype Using Simulation. SAE Technical Paper 2016-01-0167.

Le Good, G., Resnick, M., Boardman, P., Clough, B. (2018). Effects on the Aerodynamic Characteristics of Vehicles in Longitudinal Proximity Due to Changes in Style. SAE Technical Paper 2018-37-0018, pp. 1-15.

Meile, W., Brenn, G., Reppenhagen, A., Lechner, B., Fuchs, A. (2011). Experiments and numerical simulations on the aerodynamics of the Ahmed body, CFD Letters, 3(1), pp. 32-39.

Schito, P., Braghin, F. (2012). Numerical and Experimental Investigation on Vehicles in Platoon. SAE International Journal of Commercial Vehicles, 5(1), pp. 63-71.

Siemon, M., Nichols, D. S. (2018). CFD Analysis of Heterogeneous and Homogeneous Multi-Truck Platoon Aerodynamic Drag Reduction, AIAA Fluid Dynamics Conference, pp. 1-21.

Simmonds, N., Pitman, J., Tsoutsanis, P., Jenkins, K., Gaylard, A., Jansen, W. (2017). Complete body aerodynamic study of three vehicles. SAE Technical Paper 201701-1529.

Vohra, V., Wahba, M., Akarslan, G., Ni, R., Brennan, S. (2018). An examination of vehicle spacing to reduce aerodynamic drag in truck platoons, Vehicle Power and Propulsion Conference (VPPC).

Zabat, M., Frascaroli, S., Browand, F. K. (1994). Drag Measurements on 2, 3 and 4 Car Platoon. SAE Technical Paper 940421, pp. 127-138.

\begin{abstract}
.
This paper presents simulation studies on the aerodynamics of vehicles moving in an organized column. The object of research is a column that consist of three vehicles of the same type (homogeneous column). In this research geometry of Ford Transit was used. As a part of the studies, the air drag forces acting on individual vehicles were calculated. The results are presented in dimensionless drag coefficient. The influence of the distance between cars on the generated force was also determined. In the first stage of the work, a numerical model was developed based on the Ahmed body reference structure. The calculations were carried out for 9 different velocities. The obtained results of the drag coefficient were compared with the work of other authors. The applied turbulence model and parameters of the boundary layer were used to create a numerical model of a moving column of vehicles. Mesh independence for numerical model of van was verified. The Finite Volume Method was implemented in the ANSYS Fluent program and used for the calculations. The use of supercomputers was necessary due to the large size of the grid.
\end{abstract}

Keywords: aerodynamics, platooning, RANS, CFD, vehicles 